\title{
Sutured Heegaard diagrams for knots
}

\author{
YI NI
}

\begin{abstract}
We define sutured Heegaard diagrams for null-homologous knots in 3-manifolds. These diagrams are useful for computing the knot Floer homology at the top filtration level. As an application, we give a formula for the knot Floer homology of a Murasugi sum. Our result echoes Gabai's earlier works. We also show that for socalled "semifibred" satellite knots, the top filtration term of the knot Floer homology is isomorphic to the counterpart of the companion.
\end{abstract}

57R58, 57M27; 53D40

\section{Introduction}

Knot Floer homology was introduced by Ozsváth and Szabó in [10], and independently by Rasmussen in [14], as part of Ozsváth and Szabó's Heegaard Floer theory. A survey of Heegaard Floer theory can be found in Ozsváth and Szabó [8].

One remarkable feature of knot Floer homology is that it determines the genus in the case of classical knots (Ozsváth and Szabó [9, Theorem 1.2]), namely, the genus of a classical knot is the highest nontrivial filtration level of the knot Floer homology. The proof of this deep result uses Gabai's work on the existence of taut foliations of knot complements [5]. Another theorem of Gabai can be used to generalize Ozsváth and Szabó's result to links in homology 3-spheres (Ni [7]). Hence one may naturally expect that there is a more precise relationship between taut foliation and the top filtration term of knot Floer homology.

Another interesting property of knot Floer homology is that, for fibred knots, the top filtration term of knot Floer homology is a single $\mathbb{Z}$ (Ozsváth and Szabó [13, Theorem 1.1]). It is conjectured that the converse is also true for classical knots.

The results cited above show that a lot of information about the knot is contained in the top filtration term of knot Floer homology. In the present paper, we introduce sutured Heegaard diagrams for knots, which are useful for computing the top filtration term of knot Floer homology.

The definition of a sutured Heegaard diagram will be given in Section 2. We state here two theorems as applications: 
Theorem 1.1 Suppose $K_{1}, K_{2} \subset S^{3}$ are two knots and that $K$ is the Murasugi sum performed along the minimal genus Seifert surfaces. Suppose the genera of $K_{1}, K_{2}, K$ are $g_{1}, g_{2}, g$, respectively, then

$$
\widehat{H F K}(K, g ; \mathbb{F}) \cong \widehat{H F K}\left(K_{1}, g_{1} ; \mathbb{F}\right) \otimes \widehat{H F K}\left(K_{2}, g_{2} ; \mathbb{F}\right)
$$

as linear spaces, for any field $\mathbb{F}$.

Theorem 1.2 Suppose that $K^{*}$ is a semifibred satellite knot of $K$. Suppose the genera of $K, K^{*}$ are $g, g^{*}$, respectively. Then

$$
\widehat{H F K}\left(K^{*}, g^{*}\right) \cong \widehat{H F K}(K, g)
$$

as abelian groups.

The precise definitions of Murasugi sum and semifibred satellite knot will be given later, where we will prove more general versions of these two theorems.

The paper is organized as follows.

In Section 2 we review the adjunction inequality. Then we give the definition of a sutured Heegaard diagram.

In Section 3, we enhance Ozsváth and Szabó's winding argument. Using this argument, we show that a sutured Heegaard diagram may conveniently be used to compute the top filtration term of the knot Floer homology. As an immediate application, we give a new proof of a result due to Ozsváth and Szabó.

Section 4 will be devoted to the study of Murasugi sum. The formula for Murasugi sum is almost a direct corollary of the results in Section 3, once we know what the Heegaard diagram is. Our formula echoes Gabai's earlier works.

Section 5 is about semifibred satellite knots. Again, most efforts are put on the construction of a Heegaard diagram.

Acknowledgements We are grateful to David Gabai, Jacob Rasmussen and Zoltán Szabó for many stimulating discussions and encouragements. We are especially grateful to the referee for a detailed list of corrections and suggestions.

The author is partially supported by the Centennial fellowship of the Graduate School at Princeton University. Part of this work was carried out during a visit to Peking University; the author wishes to thank Shicheng Wang for his hospitality during the visit. 


\section{Sutured Heegaard diagrams}

The definition of a sutured Heegaard diagram relies on a detailed understanding of the adjunction inequality for knot Floer homology. For the reader's convenience, we sketch a proof here, which is derived from arguments of Ozsváth and Szabó ([11, Theorem 7.1] and [10, Theorem 5.1]).

Theorem 2.1 [11, Theorem 7.1] Let $K \subset Y$ be an oriented null-homologous knot, and suppose that $\widehat{H F K}(Y, K, \underline{\mathfrak{s}}) \neq 0$. Then, for each Seifert surface $F$ for $K$ of genus $g>0$, we have that

$$
\left|\left\langle c_{1}(\underline{\mathfrak{s}}),[\widehat{F}]\right\rangle\right| \leq 2 g(F) .
$$

Sketch of proof The proof consists of 3 steps.

Step 1 Construct a Heegaard splitting of $Y$

Consider a product neighborhood of $F$ in $Y: N(F)=F \times[0,1]$. Let

$$
\mathcal{H}=D^{1} \times D^{2} \subset Y-\operatorname{int}(N(F))
$$

be a 1-handle connecting $F \times 0$ to $F \times 1, \partial_{v} \mathcal{H}=D^{1} \times \partial D^{2}$ is the vertical boundary of $\mathcal{H}$. We can choose $\mathcal{H}$ so that it is "parallel" to point $\times[0,1]$ for a point on $\partial F$. Namely, there is a properly embedded product disk $\mathfrak{D}$ in $Y-\operatorname{int}(N(F) \cup \mathcal{H}), \partial \mathfrak{D} \cap(\partial F \times[0,1])$ is an essential arc in $\partial F \times[0,1]$, and $\partial \mathfrak{D} \cap \partial_{v} \mathcal{H}$ is an essential arc in $\partial_{v} \mathcal{H}$.

Let $M=Y-\operatorname{int}(N(F) \cup \mathcal{H} \cup N(\mathfrak{D})), f: M \rightarrow[0,3]$ be a Morse function, so that $\partial M=f^{-1}(0),\{$ Critical points of index $i\} \subset f^{-1}(i)$ for $i=0,1,2,3$. Let $\widetilde{\Sigma}=f^{-1}\left(\frac{3}{2}\right), \tilde{\Sigma}$ separates $Y$ into two parts $\widetilde{U}_{0}, \widetilde{U}_{1}$. Suppose $\widetilde{U}_{0}$ is the part containing $F$, then $\widetilde{U}_{0}$ can be obtained by adding $r$ 1-handles to $N(F) \cup \mathcal{H} \cup N(\mathfrak{D})$. After handlesliding, one can assume these 1-handles are attached to $F \times 0$. Moreover, we can let the two feet of any 1 -handle be very close on $F \times 0$.

$\tilde{U}_{1}$ is a handlebody, and $N(\mathfrak{D})$ is a 1-handle attached to it, if you turn $\tilde{U}_{0} \cup \tilde{U}_{1}$ upsidedown. Now let $U_{1}=\widetilde{U}_{1} \cup N(\mathfrak{D}), U_{0}=Y-\operatorname{int}\left(U_{1}\right)$, then $Y=U_{0} \cup_{\Sigma} U_{1}$ is a Heegaard splitting of $Y$.

Step 2 Find a weakly admissible Heegaard diagram for $(Y, K)$

For each 1-handle $D^{1} \times D^{2}$ attached to $F \times[0,1]$, choose its belt circle $0 \times \partial D^{2}$ $\left(D^{1}\right.$ viewed as $\left.[-1,1]\right), \alpha_{1}$ denotes the belt circle of $\mathcal{H}$, and the belt circles of other 1 -handles are denoted by $\alpha_{2 g+2}, \ldots, \alpha_{2 g+1+r}$. 
Choose a set of disjoint, properly embedded $\operatorname{arcs} \xi_{i}(i=2,3, \ldots, 2 g+1)$ on $F \times 1$, so that they represent a basis of $\mathrm{H}_{1}(F, \partial F)$. Choose a copy of $\xi_{i}$ on $F \times 0$, denoted by $\bar{\xi}_{i}$. For each $i$, complete $\xi_{i} \sqcup \bar{\xi}_{i}$ by two vertical arcs on $\partial F \times[0,1]$ to get a simple closed curve $\alpha_{i}$ on $\partial(F \times[0,1])$, which bounds a disk in $F \times[0,1] .\left(\alpha_{i}\right.$ can be viewed as the "double" of $\xi_{i}$.) We can choose $\xi_{i}$ so that $\alpha_{i}$ is disjoint from $\partial \mathfrak{D}, i=2, \ldots, 2 g+1$.

Let $\lambda=\partial F \times 1$ be the longitude of $K$, and $\mu=\partial \mathfrak{D}$ will be the meridian of $K$. Both $\lambda$ and $\mu$ are simple closed curves on $\Sigma$. Extend $\mu$ to a set of disjoint simple closed curves $\left\{\mu, \beta_{2}, \beta_{3}, \ldots, \beta_{2 g+1+r}\right\}$ on $\Sigma$, so that they are linearly independent in $\mathrm{H}_{1}(\Sigma)$, and each bounds a non-separating disk in $U_{1}$. Let $\boldsymbol{\alpha}=\left\{\alpha_{1}, \ldots, \alpha_{2 g+1+r}\right\}, \boldsymbol{\beta}_{\mathbf{0}}=$ $\left\{\beta_{2}, \ldots, \beta_{2 g+1+r}\right\}$. Then $\left(\Sigma, \boldsymbol{\alpha}, \boldsymbol{\beta}_{\mathbf{0}} \cup\{\mu\}\right)$ is a Heegaard diagram for $Y$.

Since $|\lambda \cap \mu|=1$, by handleslides over $\mu$, we can assume all $\beta_{i}$ 's $(2 \leq i \leq 2 g+1+r)$ are disjoint from $\lambda$. Now it is easy to see $\left(\Sigma, \boldsymbol{\alpha}, \boldsymbol{\beta}_{\mathbf{0}}, \mu, \lambda \cap \mu\right)$ is a marked Heegaard diagram for the knot $(Y, K)$. Furthermore, we construct a double-pointed Heegaard diagram $\left(\Sigma, \boldsymbol{\alpha}, \boldsymbol{\beta}_{\mathbf{0}} \cup\{\mu\}, w, z\right)$ from the marked one.

Choose a set of circles $\left\{\tau_{2}, \tau_{3}, \ldots, \tau_{2 g+1+r}\right\}$ on $\Sigma-\lambda-\alpha_{1}-\mu$, so that $\tau_{i}$ intersects $\alpha_{i}$ transversely and exactly once, $\tau_{i} \cap \alpha_{j}=\varnothing$ when $i \neq j$. Wind $\alpha_{i}$ 's along $\tau_{i}$ 's $(i=2, \ldots, 2 g+1+r)$, one can get a weakly admissible Heegaard diagram for $(Y, K)$. For more details, see [11, Theorem 7.1] or the discussion after Definition 3.1.

\section{Step 3 Proof of the inequality}

On $\Sigma$, there is a domain $\mathcal{P}$ bounded by $\alpha_{1}$ and $\lambda$, which is basically $F \times 1$ with a hole. Move $w, z$ slightly out of $\mathcal{P}$. Hence $\mathcal{P}$ is a periodic domain for the $Y_{0}$ Heegaard diagram $\left(\Sigma, \boldsymbol{\alpha}, \boldsymbol{\beta}_{\mathbf{0}} \cup\{\lambda\}, w\right)$.

Wind $\lambda$ once along $\mu$ as shown in Figure 1, which should be compared with [10, Figure 6]. After winding, $\lambda$ becomes a new curve $\lambda^{\prime}$, and $\mathcal{P}$ becomes $\mathcal{P}^{\prime}$. By our choice, $\mu$ has no intersection with $\xi_{i}$. Hence any intersection point $\mathbf{x}$ for the $(Y, K)$ diagram $\left(\Sigma, \boldsymbol{\alpha}, \boldsymbol{\beta}_{\mathbf{0}} \cup\{\mu\}, w, z\right)$ must contain $x_{1}=\mu \cap \alpha_{1}$. Let $\mathbf{x}^{\prime}$ be the nearby intersection for the $Y_{0}$ diagram $\left(\Sigma, \boldsymbol{\alpha}, \boldsymbol{\beta}_{\mathbf{0}} \cup\left\{\lambda^{\prime}\right\}, w\right), \mathbf{x}^{\prime}$ contains $x_{1}^{\prime}$. Here $Y_{0}$ is the manifold obtained from $Y$ by 0 -surgery on $K$. Local multiplicity of $\mathcal{P}^{\prime}$ at $x_{1}^{\prime}$ is 0 . The same argument as in the proof of [10, Theorem 5.1] shows that

$$
\left\langle c_{1}\left(\mathfrak{s}^{\prime}\left(\mathbf{x}^{\prime}\right)\right),[\widehat{F}]\right\rangle=-2 g+\#\left(x_{i} \text { in the interior of } \mathcal{P}\right) \geq-2 g .
$$

By conjugation invariance, we have the adjunction inequality.

The reader should note, in the proof of [10, Theorem 5.1], it is not assumed that $\alpha_{1}$ is the only $\alpha$ curve intersecting $\mu$. 


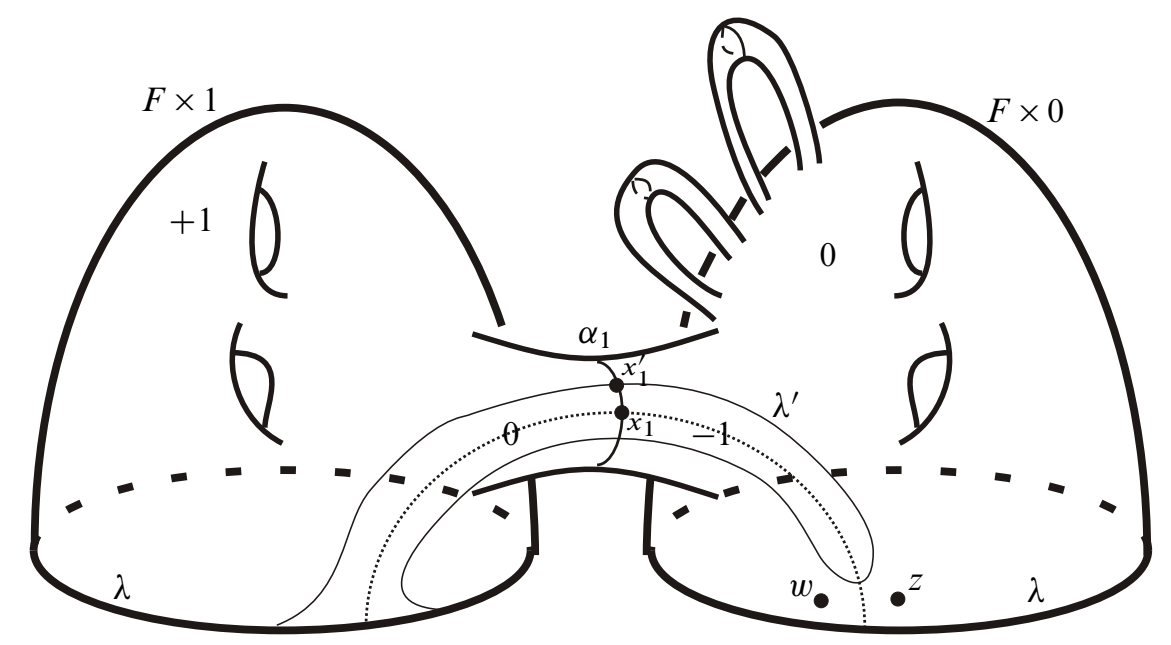

Figure 1: The Heegaard surface $\Sigma$, here the two $\lambda$ 's are glued together. Note the multiplicities of $\mathcal{P}$ in the regions on the diagram, denoted by $-1,0,+1$.

Before moving on, we clarify one convention we are going to use. The boundary map in Heegaard Floer theory is defined via counting holomorphic disks in $\operatorname{Sym}^{n} \Sigma$. There is a natural $n$-value map $\varrho: \operatorname{Sym}^{n} \Sigma \rightarrow \Sigma$. In practice we always consider the image of the holomorphic disk under $\varrho$. By abuse of notation, we do not distinguish a holomorphic disk and its image under $\varrho$.

As we have seen in the above proof, the chain complex $\widehat{C F K}(Y, K,-g)$ is generated by the intersection points

$$
\left\{\mathbf{x} \mid \text { no } x_{i} \text { lies in the interior of } \mathcal{P}\right\}
$$

for the diagram $\left(\Sigma, \boldsymbol{\alpha}, \boldsymbol{\beta}_{\mathbf{0}} \cup\{\mu\}, w, z\right)$. However, it is not clear that the holomorphic disks connecting the generators of $\widehat{C F K}(Y, K,-g)$ do not intersect the interior of $\mathcal{P}$. In fact, if two components of $\boldsymbol{\beta}_{\mathbf{0}} \cap \mathcal{P}$ are parallel, then it is very possible to have a holomorphic disk of quadrilateral type, which connects two generators of $\widehat{C F K}(Y, K,-g)$ and intersects the interior of $\mathcal{P}$.

However, if the Heegaard diagram is good enough, we can let all the holomorphic disks be supported away from $\lambda$. This observation leads to the following

Definition 2.2 A double pointed Heegaard diagram

$$
\left(\Sigma, \boldsymbol{\alpha}, \boldsymbol{\beta}_{\mathbf{0}} \cup\{\mu\}, w, z\right)
$$

for $(Y, K)$ is a sutured Heegaard diagram, if it satisfies: 
(Su0) There exists a subsurface $\mathcal{P} \subset \Sigma$, bounded by two curves $\alpha_{1} \in \boldsymbol{\alpha}$ and $\lambda . g$ denotes the genus of $\mathcal{P}$.

(Su1) $\lambda$ is disjoint from $\boldsymbol{\beta}_{\mathbf{0}} \cdot \mu$ does not intersect any $\alpha$ curves except $\alpha_{1} \cdot \mu$ intersects $\lambda$ transversely in exactly one point, and intersects $\alpha_{1}$ transversely in exactly one point. $w, z \in \lambda$ lie in a small neighborhood of $\lambda \cap \mu$, and on different sides of $\mu$. (In practice, we often push $w, z$ off $\lambda$ into $\mathcal{P}$ or $\Sigma-\mathcal{P}$.)

(Su2) $\left(\boldsymbol{\alpha}-\left\{\alpha_{1}\right\}\right) \cap \mathcal{P}$ consists of $2 g$ arcs, which are linearly independent in $\mathrm{H}_{1}(\mathcal{P}, \partial \mathcal{P})$. Moreover, $\Sigma-\boldsymbol{\alpha}-\mathcal{P}$ is connected.

The existence of a sutured Heegaard diagram is guaranteed by the construction in the proof of Theorem 2.1 .

\section{Top filtration term of the knot Floer homology}

The following definition will be useful:

Definition 3.1 In [12, Section 2.4], there are definitions of domain and periodic domain. One can generalize these definitions to relative case. For example, suppose $\mathcal{R}$ is an oriented connected compact surface, with some base points on it. $\Gamma$ is a set of finitely many properly embedded, mutually transverse curves on $\mathcal{R}$. Let $\mathcal{D}_{1}, \ldots, \mathcal{D}_{m}$ be the closures of $\mathcal{R}-\Gamma$. A relative domain $\mathcal{D}$ is a linear combination of the $\mathcal{D}_{i}$ 's. A relative periodic domain is a relative domain $\mathcal{D}$, such that $\partial \mathcal{D}-\partial \mathcal{R}$ is a linear combination of curves in $\Gamma$, and $\mathcal{D}$ avoids the basepoints on $\mathcal{R}$. If a relative periodic domain has nonnegative local multiplicities everywhere, then we call it a nonnegative relative periodic domain.

In order to get admissible diagrams, Ozsváth and Szabó introduced the technique of winding in [12]. We briefly review this technique in our relative settings.

Let $(\mathcal{R}, \Gamma)$ be as in Definition 3.1, $\xi \in \Gamma$. Suppose there exists a simple closed curve $\tau \subset \mathcal{R}$, which intersects $\xi$ transversely once. We can wind $\xi$ once along $\tau$, as shown in Figure $2 b$. Now suppose $\mathcal{D}$ is a relative periodic domain, such that $\xi$ nontrivially contributes to $\partial \mathcal{D}$, say, the contribution is 1 .

In Figure 2a, the local multiplicities of $\mathcal{D}$ in the two regions are $a$ and $a-1$, respectively. Suppose $\mathcal{D}^{\prime}$ is a variant of $\mathcal{D}$ after winding. In Figure $2 b$, the local multiplicity of $\mathcal{D}^{\prime}$ in the shaded area is $a-2$. If we wind $\xi$ along $\tau$ sufficiently many times, we can get negative local multiplicity here. If we wind $\xi$ along a parallel copy of $\tau$, but in the other direction, we can get positive local multiplicity. 


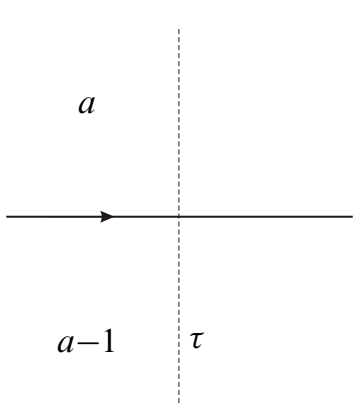

Figure 2a

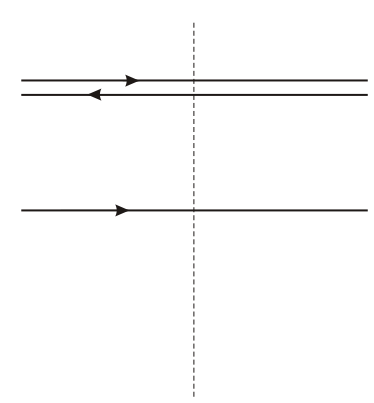

Figure 2c

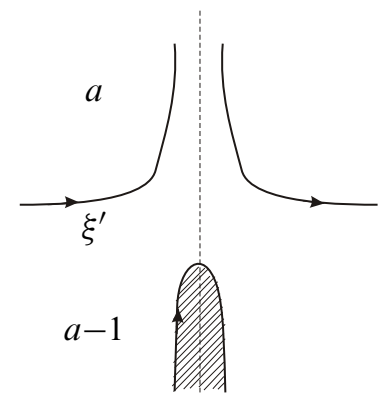

Figure $2 b$

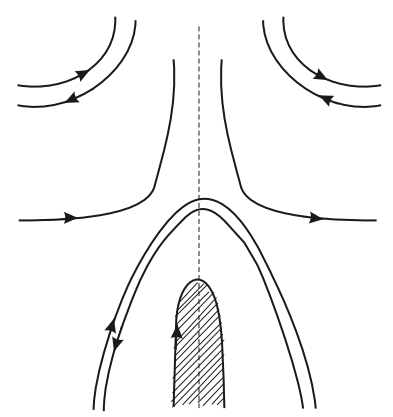

Figure 2d

Figure 2: Wind $\xi$ along $\tau$

Sometimes we have to wind several curves simultaneously along $\tau$. We require the curve $\xi$, which we care about, has nonzero algebraic intersection number with $\tau$; and all other curves we wind have zero algebraic intersection number with $\tau$. Then we can still get both positive and negative local multiplicities after winding. See Figure $2 c$ and Figure $2 \mathrm{~d}$ for a typical example.

Now we can give a key lemma:

Lemma 3.2 Suppose $\mathcal{R}$ is an oriented connected compact surface, with a nonempty collection of base points $\mathbf{w}$. Let $\Gamma$ be a set of finitely many properly embedded, mutually transverse curves on $\mathcal{R} . \Gamma=\Xi \sqcup \Theta, \Xi=\left\{\xi_{1}, \ldots, \xi_{n}\right\}$. Suppose that there are circles $\tau_{1}, \ldots, \tau_{n}$, such that $\tau_{i}$ intersects $\xi_{i}$ transversely in a single intersection point. Furthermore, suppose there exists a region (connected open set) $\mathcal{U}$, so that $\mathbf{w} \subset \mathcal{U}, \tau_{i} \cap \xi_{i} \in \mathcal{U}$ for all $i$, and all curves in $\Theta$ are disjoint from $\mathcal{U}$.

Then after winding $\xi$ curves along the $\tau$ curves sufficiently many times, every relative periodic domain $\mathcal{D}$ whose boundary contains $\xi_{i}$ nontrivially (ie, $n_{i} \cdot \xi_{i} \subset \partial \mathcal{D}, n_{i} \neq 0$ ) 
has both positive and negative local multiplicities. Hence there is no nonnegative relative period domain $\mathcal{D}$ with its boundary containing $\xi_{i}$ nontrivially.

Furthermore, if the algebraic intersection number of $\tau_{i}$ with $\xi_{j}$ is zero when $i \neq j$, and $\xi_{i}$ 's are mutually disjoint, then we can arrange that the $\xi_{i}$ 's are mutually disjoint after winding.

Proof For convenience, we use $\mathbb{Q}$ coefficients. Without loss of generality, we can assume $\mathbf{w}$ consists of a single point $w$. Let $\mathfrak{X}$ be the linear space generated by curves in $\Xi, \mathfrak{Y}$ be the linear space generated by curves in $\Theta$. There is a natural homomorphism

$$
\mathfrak{S}: \mathfrak{X} \oplus \mathfrak{Y} \rightarrow \mathrm{H}_{1}(\mathcal{R}, \partial \mathcal{R}) .
$$

For each nontrivial element $\gamma \in \operatorname{ker} \mathfrak{F}$, there is a unique relative periodic domain

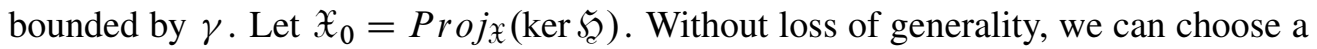
basis of $\mathfrak{X}_{0}$ in the form

$$
\zeta_{i}=\xi_{i}+\sum_{j=m+1}^{n} c_{i j} \xi_{j}, \quad i=1, \ldots, m .
$$

Each $\zeta_{i}$ cobounds a relative periodic domain $\mathcal{Q}_{i}$ with some element in $\mathfrak{Y}$.

We will wind $\xi_{i}$ along $\tau_{i}$ in one direction sufficiently many times, and wind $\xi_{i}$ along a parallel copy of $\tau_{i}$ in the other direction sufficiently many times, thus get new collection of curves $\Xi^{\prime}$. The variants of $\mathcal{Q}_{i}$ after winding are denoted by $\mathcal{Q}_{i}^{\prime}$. Then for each $i$, there are points $w_{i}, z_{i}$ near $\xi_{i} \cap \tau_{i}$ (hence $w_{i}, z_{i} \in \mathcal{U}$ ), such that $n_{w_{i}}\left(\mathcal{Q}_{i}^{\prime}\right)$ is positive, $n_{z_{i}}\left(\mathcal{Q}_{i}^{\prime}\right)$ is negative.

Note that the winding along $\tau$ only changes the local multiplicities in a neighborhood of $\tau$. We can choose those neighborhoods narrow enough, so that $w_{i}, z_{i}$ are not in the neighborhood of $\tau_{j}$ when $j \neq i$. Hence $n_{w_{i}}\left(\mathcal{Q}_{j}^{\prime}\right)=n_{w_{i}}\left(\mathcal{Q}_{j}\right)$ when $j \neq i$. We wind $\xi_{i}$ sufficiently many times, so that

$$
n_{w_{i}}\left(\mathcal{Q}_{i}^{\prime}\right)>\sum_{j \neq i}\left|n_{w_{i}}\left(\mathcal{Q}_{j}\right)\right|, \quad\left|n_{z_{i}}\left(\mathcal{Q}_{i}^{\prime}\right)\right|>\sum_{j \neq i}\left|n_{z_{i}}\left(\mathcal{Q}_{j}\right)\right| .
$$

Now if $\mathcal{D}$ is a relative periodic domain, and $\partial \mathcal{D}$ contains some $\xi_{i}^{\prime}$ nontrivially, then $\operatorname{Proj}_{\mathfrak{X}}(\partial \mathcal{D})=\sum_{i=1}^{m} c_{i} \zeta_{i}^{\prime}$ is a nontrivial sum. $\mathcal{D}-\sum_{i=1}^{m} c_{i} \mathcal{Q}_{i}^{\prime}$ is a relative periodic domain bounded by an element in $\mathfrak{Y}$. But all curves generating $\mathfrak{Y}$ are disjoint from $\mathcal{U} \ni w$, so $\mathcal{D}-\sum_{i=1}^{m} c_{i} \mathcal{Q}_{i}^{\prime}$ is disjoint from $\mathcal{U}$. Suppose $c_{l}$ is the coefficient with maximal absolute value, then $\mathcal{D}$ has negative multiplicity at $w_{l}$ or $z_{l}$.

If the algebraic intersection number of $\tau_{i}$ with $\xi_{j}$ is zero when $i \neq j$, and $\xi_{i}$ 's are mutually disjoint before winding, then when we wind along $\tau_{i}$, we simultaneously 
wind all the $\xi$ curves intersecting $\tau_{i}$. Hence $\xi_{i}$ 's are still disjoint after winding. We can get our result about local multiplicity by the discussion before Lemma 3.2.

Suppose $\left(\Sigma, \boldsymbol{\alpha}, \boldsymbol{\beta}_{\mathbf{0}} \cup\{\mu\}, w, z\right)$ is a sutured Heegaard diagram for $(Y, K)$. As in the proof of Theorem 2.1, the generators of $\widehat{C F K}(Y, K,-g)$ are supported outside the interior of $\mathcal{P}$. Our main result is

Proposition 3.3 Let $\left(\Sigma, \boldsymbol{\alpha}, \boldsymbol{\beta}_{\mathbf{0}} \cup\{\mu\}, w, z\right)$ be a sutured Heegaard diagram for $(Y, K)$. Then after winding transverse to the $\alpha$ curves, we get a new sutured Heegaard diagram

$$
\left(\Sigma, \boldsymbol{\alpha}^{\prime \prime}, \boldsymbol{\beta}_{\mathbf{0}} \cup\{\mu\}, w, z\right),
$$

which is weakly admissible, and all the holomorphic disks connecting generators of $\widehat{C F K}(Y, K,-g)$ are supported outside a neighborhood of $\lambda$.

Proof Suppose the components of $\left(\boldsymbol{\alpha}-\left\{\alpha_{1}\right\}\right) \cap \mathcal{P}$ are $\xi_{2}, \ldots, \xi_{2 g+1}$. Let $\mathcal{U}$ be a small neighborhood of $\lambda$.

$\xi_{2}, \ldots, \xi_{2 g+1}$ are linearly independent in $\mathrm{H}_{1}(\mathcal{P}, \partial \mathcal{P})$, and they are disjoint from $\mu \cap \mathcal{P}$, which is an arc connecting $\alpha_{1}$ to $\lambda$. Hence $\mathcal{P}-\cup_{i=2}^{2 g+1} \xi_{i}-\mu$ is connected. Now we can find simple closed curves $\tau_{2}, \ldots, \tau_{2 g+1} \subset \mathcal{P}$, so that they are disjoint from $\mu$ and $\xi$ curves, except that $\tau_{i}$ intersects $\xi_{i}$ transversely in a single intersection point. We can assume $\tau_{i} \cap \xi_{i} \in \mathcal{U}$. By Lemma 3.2, after winding $\xi$ curves along $\tau$ curves, we can get a new Heegaard diagram

$$
\left(\Sigma, \boldsymbol{\alpha}^{\prime}, \boldsymbol{\beta}_{\mathbf{0}} \cup\{\mu\}, w, z\right),
$$

such that its restriction to $\mathcal{P}$ satisfies the conclusion of Lemma 3.2.

Suppose the closed $\alpha$ curves in $\Sigma-\mathcal{P}$ are $\widetilde{\alpha}_{2 g+2}, \ldots, \widetilde{\alpha}_{2 g+1+r}$. By Condition (Su2), $\Sigma-\boldsymbol{\alpha}-\mathcal{P}$ is connected, hence we can find circles $\tau_{2 g+2}, \ldots, \tau_{2 g+1+r} \subset(\Sigma-\mathcal{P})$, such that they are disjoint from all the $\alpha$ curves, except that $\tau_{i}$ intersects $\widetilde{\alpha}_{i}$ transversely in a single intersection point.

Now if $\mathcal{D}$ is a periodic domain, then $\mathcal{D} \cap \mathcal{P}$ is a relative periodic domain in $\mathcal{P}$. Hence if $\mathcal{D}$ is non-negative, then $\partial \mathcal{D}$ does not pass through $\xi_{2}, \ldots, \xi_{2 g+1}$. A similar argument as in the proof of Lemma 3.2 shows that we can wind $\widetilde{\alpha}_{2 g+2}, \ldots, \widetilde{\alpha}_{2 g+1+r}$ along $\tau_{2 g+2}, \ldots, \tau_{2 g+1+r}$, to get a new diagram

$$
\left(\Sigma, \boldsymbol{\alpha}^{\prime \prime}, \boldsymbol{\beta}_{\mathbf{0}} \cup\{\mu\}, w, z\right),
$$

such that $\partial \mathcal{D}$ does not pass through $\widetilde{\alpha}_{2 g+2}, \ldots, \widetilde{\alpha}_{2 g+1+r}$.

So $\partial \mathcal{D}$ consists of $\alpha_{1}$ and curves in $\boldsymbol{\beta}_{\mathbf{0}}$. Moreover, consider the curve $\mu$. We observe that the base points $w, z$ lie close to and on both sides of $\mu$, and $\mu$ has only one 
intersection with the $\alpha$ curves. So $\mathcal{D} \cap \mu=\varnothing . \mu$ intersects $\alpha_{1}$, hence $\partial \mathcal{D}$ does not contain $\alpha_{1}$. We conclude that $\left(\Sigma, \boldsymbol{\alpha}^{\prime \prime}, \boldsymbol{\beta}_{\mathbf{0}} \cup\{\mu\}, w, z\right)$ is weakly admissible, since curves in $\boldsymbol{\beta}_{\mathbf{0}}$ are linearly independent in $\mathrm{H}_{1}(\Sigma)$.

If $\Phi$ is a holomorphic disk connecting two generators of $\widehat{C F K}(Y, K,-g)$, then $\Phi \cap \mathcal{P}$ is a relative periodic domain in $\mathcal{P}$, since the generators lie outside $\operatorname{int}(\mathcal{P})$. So $\partial \Phi$ does not pass through $\xi$ curves. Hence $\Phi$ is disjoint from $\lambda$, since it should avoid $w, z$.

Remark 3.4 In practice, in order to compute $\widehat{H F K}(Y, K,-g)$ from a given sutured Heegaard diagram, we only need to wind the closed $\alpha$ curves in $\Sigma-\mathcal{P}$ sufficiently many times, then count the holomorphic disks which are disjoint from $\lambda$. The reason is that these disks are not different from those disks obtained after winding $\xi$ curves.

As an immediate application, we give the following proposition. This proposition and the second proof here were told to the author by Zoltán Szabó. The current paper was partially motivated by an attempt to understand this proposition.

Proposition 3.5 $(Y, K)$ is a knot, $F$ is its Seifert surface of genus $g$. We cut open $Y$ along $F$, then reglue by a self-diffeomorphism $\varphi$ of $F$. Denote the new knot in the new manifold by $\left(Y^{\prime}, K^{\prime}\right)$. Then

$$
\widehat{H F K}(Y, K,-g) \cong \widehat{H F K}\left(Y^{\prime}, K^{\prime},-g\right)
$$

as abelian groups.

The first proof Construct a sutured Heegaard diagram $\left(\Sigma, \boldsymbol{\alpha}, \boldsymbol{\beta}_{\mathbf{0}} \cup\{\mu\}, w, z\right)$ from $F$, as in the proof of Theorem 2.1. We can assume this diagram already satisfies the conclusion of Proposition 3.3. The subsurface $\mathcal{P} \subset \Sigma$ is more or less a punctured $F$. We can extend $\varphi$ by identity to a diffeomorphism of $\Sigma$. $\left(Y^{\prime}, K^{\prime}\right)$ has Heegaard diagram $\left(\Sigma, \varphi(\boldsymbol{\alpha}), \boldsymbol{\beta}_{\mathbf{0}} \cup\{\mu\}, w, z\right)$. The generators of $\widehat{C F K}(Y, K,-g)$ and $\widehat{C F K}\left(Y^{\prime}, K^{\prime},-g\right)$ are the same. It is easy to see the boundary maps are also the same, since the boundary of a holomorphic disk does not pass through $\alpha$ curves inside $\mathcal{P}$.

The second proof Suppose $\gamma$ is a circle in $F$, with a framing induced by $F$. Since $\gamma$ can be isotoped off $F$, we have the surgery exact triangle (see [10, Theorem 8.2]):

$\cdots \rightarrow \widehat{H F K}\left(Y_{-1}(\gamma), K,-g\right) \rightarrow \widehat{H F K}\left(Y_{0}(\gamma), K,-g\right) \rightarrow \widehat{H F K}(Y, K,-g) \rightarrow \cdots$.

$\left(Y_{0}(\gamma), K\right)$ has a Seifert surface with genus $<g$, which is obtained by surgering $F$ along $\gamma$. By the adjunction inequality, $\widehat{H F K}\left(Y_{0}(\gamma), K,-g\right)=0$. Hence our result holds when $\varphi$ is the positive Dehn twist along $\gamma$. The result also holds when $\varphi$ is a negative Dehn twist, since a negative Dehn twist is just the inverse of a positive one. The general case follows since every self-diffeomorphism of $F$ is the product of Dehn twists. 


\section{Murasugi sum}

In Gabai's theory of sutured manifold decomposation, the longitude $\lambda$ of a knot often serves as the suture (see [5]). So Proposition 3.3 says that the boundary map of $\widehat{C F K}(Y, K,-g)$ "avoids the suture". This justifies the name "sutured Heegaard diagram". Using sutured Heegaard diagrams, we can give a formula for Murasugi sum. This is our first attempt to apply our method to sutured manifold decomposition.

Definition 4.1 $F^{(k)}$ is an oriented compact surface in the manifold $Y^{(k)}, k=1,2$. $B^{(k)} \subset Y^{(k)}$ is a 3-ball, $\operatorname{int}\left(B^{(k)}\right) \cap F^{(k)}=\varnothing, \partial B^{(k)} \cap F^{(k)}$ is a disk $D^{(k)}$, and $D^{(k)} \cap \partial F^{(k)}$ consists of $n$ disjoint arcs. Remove $\operatorname{int}\left(B^{(k)}\right)$ from $Y^{(k)}$, glue the punctured $Y^{(1)}$ and punctured $Y^{(2)}$ together by a homeomorphism of the boundaries, so that $D^{(1)}$ is identified with $D^{(2)}$, and $\partial D^{(1)} \cap \partial F^{(1)}$ is identified with the closure of $\partial D^{(2)}-\partial F^{(2)}$. We then get a new manifold $Y=Y^{(1)} \# Y^{(2)}$ and a surface $F=$ $F^{(1)} \cup F^{(2)}$. Then $F$ is called the Murasugi sum of $F^{(1)}$ and $F^{(2)}$, denoted by $F^{(1)} * F^{(2)}$.

$L^{(k)}=\partial F^{(k)}$ is an oriented link in $Y^{(k)}$. Then $L=\partial F$ is called the Murasugi sum of $L^{(1)}$ and $L^{(2)}$, denoted by $L^{(1)} * L^{(2)}$.

When $n=1$, this operation is merely connected sum; when $n=2$, this operation is also known as "plumbing".

Gabai showed that Murasugi sum is a natural geometric operation in [3] and [4]. We summarize some of his results here:

Theorem 4.2 (Gabai) With notation as above, we have:

(i) $F$ is a Seifert surface with maximal Euler characteristic for $L$, if and only if $F^{(1)}$ and $F^{(2)}$ are Seifert surfaces with maximal Euler characteristic for $L^{(1)}$ and $L^{(2)}$, respectively.

(ii) $Y-L$ fibers over $S^{1}$ with fiber $F$, if and only if $Y^{(k)}-L^{(k)}$ fibers over $S^{1}$ with fiber $F$ for $k=1,2$.

Our result about Murasugi sum is an analogue of Gabai's theorem in the world of knot Floer homology. We first consider the case of knots.

Proposition 4.3 Suppose knot $(Y, K)$ is the Murasugi sum of two knots $\left(Y^{(1)}, K^{(1)}\right)$ and $\left(Y^{(2)}, K^{(2)}\right)$. Genera of $F, F^{(1)}, F^{(2)}$ are $g, g^{(1)}, g^{(2)}$, respectively. Then

$$
\widehat{C F K}(Y, K,-g) \cong \widehat{C F K}\left(Y^{(1)}, K^{(1)},-g^{(1)}\right) \otimes \widehat{C F K}\left(Y^{(2)}, K^{(2)},-g^{(2)}\right)
$$


as ungraded chain complexes. In particular, for any field $\mathbb{F}$,

$$
\widehat{H F K}(Y, K,-g ; \mathbb{F}) \cong \widehat{H F K}\left(Y^{(1)}, K^{(1)},-g^{(1)} ; \mathbb{F}\right) \otimes \widehat{H F K}\left(Y^{(2)}, K^{(2)},-g^{(2)} ; \mathbb{F}\right)
$$

as linear spaces.

Proof The proof consists of 3 steps. First of all, starting from the surfaces, we construct a Heegaard splitting for the pair $(Y, K)$. Secondly, we explicitly give the $\alpha$ and $\beta$ curves on the Heegaard surface, hence we get a Heegaard diagram. This diagram is a sutured Heegaard diagram. Finally, based on the diagram, we prove our desired formula by using Proposition 3.3.

\section{Step 1 Construct a Heegaard splitting for $(Y, K)$}

$Y$ is separated into two parts $Y^{(1)}-\operatorname{int}\left(B^{(1)}\right)$ and $Y^{(2)}-\operatorname{int}\left(B^{(2)}\right)$. Let $D=F^{(1)} \cap$ $F^{(2)}$ be a $2 n$-gon. Thicken $F$ to $F \times[0,1]$ in $Y$. Add a 1 -handle $\mathcal{H}$ to connect $D \times 0$ to $D \times 1$, so that there is a simple closed curve $\mu \subset \partial((F \times[0,1]) \cup \mathcal{H})$, which bounds a disk in $Y-((F \times[0,1]) \cup \mathcal{H})$, and passes through $\mathcal{H}$ once.

Add $r^{(k)} 1$-handles $\mathcal{H}_{2 g+2}^{(k)}, \ldots, \mathcal{H}_{2 g+1+r^{(k)}}^{(k)}$ in $Y^{(k)}-B^{(k)}$ to $F^{(k)} \times 0$, as when we construct the Heegaard splitting of $\left(Y^{(k)}, K^{(k)}\right)$. After handlesliding, we can assume each 1-handle is added to a connected component of $(F-D) \times 0$. Let $U_{0}$ be the handlebody obtained by adding the $1+r^{(1)}+r^{(2)} 1$-handles to $F \times[0,1] . Y-\operatorname{int}\left(U_{0}\right)$ is also a handlebody, we hence get a Heegaard splitting for $(Y, K)$.

\section{Step 2 Construct a sutured Heegaard diagram}

$F$ is the Murasugi sum of $F^{(1)}$ and $F^{(2)}$, hence $\chi(F)=\chi\left(F^{(1)}\right)+\chi\left(F^{(2)}\right)-1$. Since $K, K^{(1)}, K^{(2)}$ are all knots, we have $g=g^{(1)}+g^{(2)}$.

We have the Mayer-Vietoris sequence:

$$
\begin{aligned}
0 & \rightarrow \mathrm{H}_{2}\left(F^{(k)}, \partial F^{(k)}\right) \rightarrow \oplus^{n} \mathrm{H}_{1}(I, \partial I) \\
& \rightarrow \mathrm{H}_{1}\left(D, D \cap \partial F^{(k)}\right) \oplus \mathrm{H}_{1}\left(F^{(k)}-D,\left(F^{(k)}-D\right) \cap \partial F^{(k)}\right) \\
& \rightarrow \mathrm{H}_{1}\left(F^{(k)}, \partial F^{(k)}\right) \rightarrow 0 .
\end{aligned}
$$

Here $I$ denotes a segment. It follows that

$$
\operatorname{rank} \mathrm{H}_{1}\left(F^{(k)}-D,\left(F^{(k)}-D\right) \cap \partial F^{(k)}\right)=\operatorname{rank} \mathrm{H}_{1}\left(F^{(k)}, \partial F^{(k)}\right),
$$

and the map

$$
\mathrm{H}_{1}\left(F^{(k)}-D,\left(F^{(k)}-D\right) \cap \partial F^{(k)}\right) \rightarrow \mathrm{H}_{1}\left(F^{(k)}, \partial F^{(k)}\right)
$$


is injective. Hence we can choose $2 g^{(k)}$ disjoint $\operatorname{arcs} \xi_{2}^{(k)}, \ldots, \xi_{2 g^{(k)}+1}^{(k)} \subset F^{(k)}-D$ representing a basis of $\mathrm{H}_{1}\left(F^{(k)}, \partial F^{(k)}\right)$. We choose the arcs such that they do not separate the two feet of any 1 -handle added to $(F-D) \times 0$. Hence for each 1 -handle $\mathcal{H}_{j}^{(k)}$ added to $(F-D) \times 0$, there is a simple closed curve

$$
\tau_{j}^{(k)} \subset \partial\left(((F-D) \times[0,1]) \cup \mathcal{H}_{j}^{(k)}\right)-(F-D) \times 1,
$$

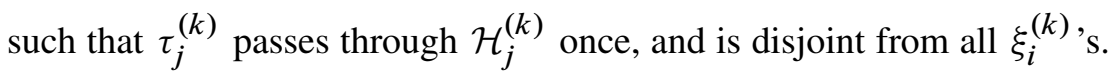

Construct a sutured Heegaard diagram

$$
\left(\Sigma^{(k)}, \boldsymbol{\alpha}^{(k)}, \boldsymbol{\beta}_{\mathbf{0}}{ }^{(k)} \cup\left\{\mu^{(k)}\right\}, w^{(k)}, z^{(k)}\right)
$$

for $\left(Y^{(k)}, K^{(k)}\right)$ as in the proof of Theorem 2.1. Here $\boldsymbol{\alpha}^{(k)}$ consists of $\alpha_{i}^{(k)}$,s and $\widetilde{\alpha}_{j}^{(k)}$ 's. $\alpha_{i}^{(k)}$ is basically the "double" of $\xi_{i}^{(k)}$ when $2 \leq i \leq 2 g^{(k)}+1$, and $\tilde{\alpha}_{j}^{(k)}$ is the belt circle of $\mathcal{H}_{j}^{(k)}$.

We can assume $\alpha_{1}^{(k)} \subset D$ is the boundary of a smaller $2 n$-gon concentric to $D$, and the restrictions of $\boldsymbol{\beta}_{\mathbf{0}}$ curves in $D \times\{0,1\}$ are segments perpendicular to the corresponding edges of $D$. Moreover, let the relative positions of $\mu^{(1)} \cap D$ and $\mu^{(2)} \cap D$ in $D$ be the same. See Figure 3 for the case when $D$ is a square.

Suppose the sides of $D$ are $a_{1}, b_{1}, a_{2}, b_{2}, \ldots, a_{n}, b_{n}$ in cyclic order, where $a_{i} \subset$ $\partial F^{(1)}, b_{i} \subset \partial F^{(2)}$. In $\Sigma^{(k)}$, there is a subsurface $Q^{(k)}$, which is the union of a punctured $D \times 0$, a punctured $D \times 1$ and a tube whose belt circle is $\alpha_{1}^{(k)}$. We glue $\Sigma^{(1)}-\cup_{i=1}^{n} \operatorname{int}\left(a_{i} \times[0,1]\right)$ and $\Sigma^{(2)}-\cup_{i=1}^{n} \operatorname{int}\left(b_{i} \times[0,1]\right)$ together, such that $Q^{(1)}$ is identified with $Q^{(2)}$, and the edge $\left(a_{i} \cap b_{i \pm 1}\right) \times[0,1]$ in $\Sigma^{(1)}$ is glued to the $\left(a_{i} \cap b_{i \pm 1}\right) \times[0,1]$ in $\Sigma^{(2)}$.

After the gluing, we get a surface $\Sigma$. We also identify $\alpha_{1}^{(1)}$ with $\alpha_{1}^{(2)}, \mu^{(1)}$ with $\mu^{(2)}$, $\left(w^{(1)}, z^{(1)}\right)$ with $\left(w^{(2)}, z^{(2)}\right)$. The objects after identification are called $Q, \alpha_{1}, \mu$, $(w, z)$, respectively.

Now we have a diagram

$$
\left(\Sigma, \boldsymbol{\alpha}^{(1)} \cup \boldsymbol{\alpha}^{(2)}, \boldsymbol{\beta}_{\mathbf{0}}{ }^{(1)} \sqcup \boldsymbol{\beta}_{\mathbf{0}}{ }^{(2)} \cup\{\mu\}, w, z\right)
$$

We can fit the curves $\boldsymbol{\alpha}^{(1)} \cup \boldsymbol{\alpha}^{(2)}, \boldsymbol{\beta}_{\mathbf{0}}{ }^{(1)} \sqcup \boldsymbol{\beta}_{\mathbf{0}}{ }^{(2)} \cup\{\mu\}$ into the Heegaard splitting we got in Step 1, so that each curve bounds a disk in some handlebody. Now it is easy to see

$$
\left(\Sigma, \boldsymbol{\alpha}^{(1)} \cup \boldsymbol{\alpha}^{(2)}, \boldsymbol{\beta}_{\mathbf{0}}{ }^{(1)} \sqcup \boldsymbol{\beta}_{\mathbf{0}}^{(2)} \cup\{\mu\}, w, z\right)
$$




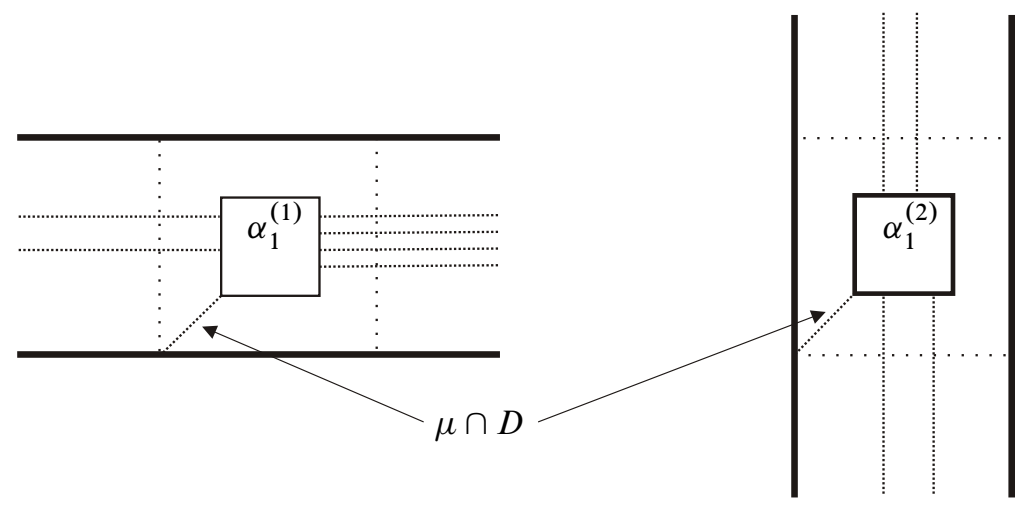

Figure 3: Local pictures of $D^{(k)} \times\{0,1\}$

is a sutured Heegaard diagram for $(Y, K)$. It is understood that the "suture" is the longitude $\lambda$ of $K$.

\section{Step 3 Prove the formula}

By Proposition 3.3 and Remark 3.4, in order to compute $\widehat{H F K}(Y, K,-g)$, we only need to wind $\widetilde{\alpha}_{j}^{(k)}$ along $\tau_{j}^{(k)}$ many times, and count the holomorphic disks which are disjoint from $\lambda$. Suppose $\Phi$ is such a disk, then the local multiplicities of $\Phi$ at the vertices of $D \times\{0,1\}$ are all 0 . As in Figure 4, we now find that $\Phi \cap Q$ is separated into two disjoint parts, one is extended into $\Sigma^{(1)}-Q$, the other is extended into $\Sigma^{(2)}-Q$. (Each part itself may be disconnected or empty.)

Since $Q$ is the only common part of $\Sigma^{(1)}$ and $\Sigma^{(2)}$, we now conclude that $\Phi$ consists of two disjoint parts, one is a holomorphic disk $\Phi^{(1)}$ in $\Sigma^{(1)}$, the other is a holomorphic $\operatorname{disk} \Phi^{(2)}$ in $\Sigma^{(2)}$. $\Phi^{(k)}$ is a holomorphic disk for $\widehat{C F K}\left(Y^{(k)}, K^{(k)},-g^{(k)}\right)$.

Conversely, if we have holomorphic disks $\Phi^{(k)}$ for $\widehat{C F K}\left(Y^{(k)}, K^{(k)},-g^{(k)}\right), k=1,2$, then they are disjoint in $\Sigma$, since they are disjoint from $\lambda^{(k)}$. Now we can put them together to get a holomorphic disk $\Phi$ for $\widehat{C F K}(Y, K,-g)$.

Now the formula is obvious.

Before dealing with the case of links, we recall the definition of knot Floer homology for links. In [10], Ozsváth and Szabó gave a well-defined correspondence from links to knots, and the homology for links is defined to be the homology for the corresponding knots.

The construction is described as follows: given any null-homologous oriented $n-$ component link $L$ in $Y$, choose two points $p, q$ on different components of $L$. Remove 


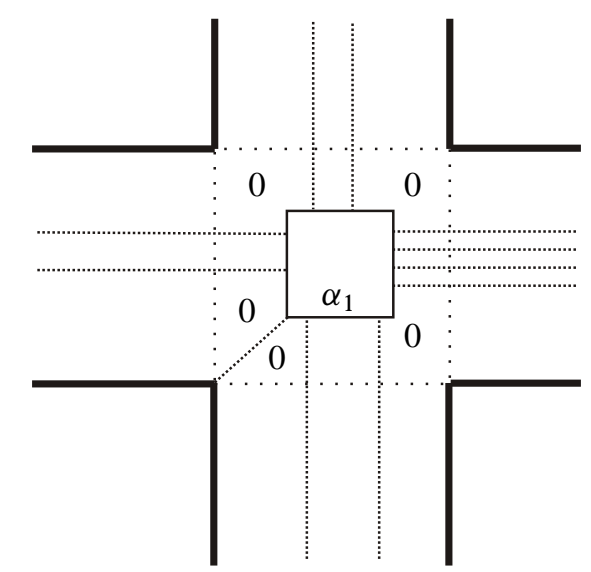

Figure 4: Local pictures of $D \times\{0,1\}$ after the operation

two balls at $p, q$, then glue in $S^{2} \times I$. Inside $S^{2} \times I$, there is a band, along which we can perform a connected sum of the two components of L containing $p$ and $q$. We choose the band so that the connected sum respects the original orientation on $L$. Now we have a link in $Y^{3} \# S^{2} \times S^{1}$, with one fewer component. Repeat this construction until we get a knot. The new knot is denoted by $\kappa(L)$, and the new manifold is denoted by $\kappa(Y)=Y \#(|L|-1)\left(S^{2} \times S^{1}\right)$. Ozsváth and Szabó proved that this correspondence $(Y, L) \mapsto(\kappa(Y), \kappa(L))$ is well-defined.

Define $\Pi$ to be a link in $S^{2} \times S^{1}$, such that $\Pi$ consists of two copies of point $\times S^{1}$, but with different orientations. $\Pi$ is a fibred link, its fiber is an annulus. When we do plumbing of $\Pi$ with other links, we always choose the annulus as the Seifert surface for $\Pi$. It is not hard to see that Ozsváth and Szabó's construction is more or less doing plumbing with copies of $\Pi$. (See [7] for an explanation.)

The next lemma is a special case of our general theorem about Murasugi sum.

Lemma 4.4 Suppose $(Y, L)$ is a link with Seifert surface $F$. Do plumbing for $L$ and $\Pi$, we get a link $\left(Y^{\prime}=Y \# S^{2} \times S^{1}, L^{\prime}\right)$ with Seifert surface $F^{\prime}$. Then

$$
\widehat{H F K}\left(Y, L, \frac{|L|-\chi(F)}{2}\right) \cong \widehat{H F K}\left(Y^{\prime}, L^{\prime}, \frac{\left|L^{\prime}\right|-\chi\left(F^{\prime}\right)}{2}\right)
$$

as abelian groups.

Proof If the plumbing merges two components of $L$, then the result holds by the discussion before this lemma. Now we consider the case that the plumbing splits a component of $L$ into two components. Without loss of generality, we can assume $L$ is a knot. 
Now $L^{\prime}=L * \Pi$ is a two-component link, we have to consider the knot $\kappa\left(L^{\prime}\right)$. But $\kappa\left(L^{\prime}\right)$ is just the plumbing of $L$ with the fibred knot $\Pi * \Pi$. Hence the result holds by Proposition 4.3.

Now we can give our general theorem about Murasugi sum.

Theorem 4.5 Notations as in Definition 4.1. Let $\mathfrak{i}(F)=\frac{|\partial F|-\chi(F)}{2}$. Given a field $\mathbb{F}$, we have

$$
\widehat{H F K}(Y, L, \mathfrak{i}(F)) \cong \widehat{H F K}\left(Y^{(1)}, L^{(1)}, \mathfrak{i}\left(F^{(1)}\right)\right) \otimes \widehat{H F K}\left(Y^{(2)}, L^{(2)}, \mathfrak{i}\left(F^{(2)}\right)\right)
$$

as linear spaces. Here we use $\mathbb{F}$-coefficients.

Proof Suppose the Murasugi sum is done along a $2 n$-gon $D$. The sides of $D$ are denoted by $a_{1}, b_{1}, a_{2}, b_{2}, \ldots, a_{n}, b_{n}$ in cyclic order, where $a_{i} \subset \partial F^{(1)}, b_{i} \subset \partial F^{(2)}$.

Push a neighborhood of $b_{i}$ slightly out of $D$, to get a rectangle $R\left(b_{i}\right)$. We do plumbing of $F^{(1)}$ with $n-1$ copies of $\Pi$, along $R\left(b_{1}\right), R\left(b_{2}\right), \ldots, R\left(b_{n-1}\right)$. We get a new link $L_{1}^{(1)}$ with Seifert surface $F_{1}^{(1)}$. There is an arc $a \subset L_{1}^{(1)}, a$ contains $a_{1}, \ldots, a_{n}$ in order. Lemma 4.4 shows that

$$
\widehat{H F K}\left(L_{1}^{(1)}, \mathfrak{i}\left(F_{1}^{(1)}\right)\right) \cong \widehat{H F K}\left(L^{(1)}, \mathfrak{i}\left(F^{(1)}\right)\right) .
$$

(We suppress the ambient 3-manifolds in the formula.)

Similarly, we plumb $F^{(2)}$ with $n-1$ copies of $\Pi$, along $R\left(a_{2}\right), R\left(a_{3}\right), \ldots, R\left(a_{n}\right)$, to get a link $L_{1}^{(2)}$. There is an $\operatorname{arc} b \subset L_{1}^{(2)}, b$ contains $b_{1}, \ldots, b_{n}$ in order. Moreover,

$$
\widehat{H F K}\left(L_{1}^{(2)}, \mathfrak{i}\left(F_{1}^{(2)}\right)\right) \cong \widehat{H F K}\left(L^{(2)}, \mathfrak{i}\left(F^{(2)}\right)\right) \text {. }
$$

We perform the operation $\kappa$ to $L_{1}^{(1)}, L_{1}^{(2)}$, with all the connecting bands added outside $a, b$, to get new knots $K^{(1)}, K^{(2)} . K^{(1)}$ contains $a_{1}, \ldots, a_{n}$ in cyclic order, and $K^{(2)}$ contains $b_{1}, \ldots, b_{n}$ in cyclic order.

Now it is easy to see the Murasugi sum $K=K^{(1)} * K^{(2)}$ is still a knot. Our result holds by Proposition 4.3 and Lemma 4.4.

\section{Semifibred satellite knots}

The reader should note that some notations in this section are different from the last section. This is not very disturbing, since this section is independent of the last one. 
Definition 5.1 Suppose $K$ is a null-homologous knot in $Y, F$ is a Seifert surface of $K$ (not necessarily has minimal genus). $V$ is a 3-manifold, $\partial V=T^{2}, L \subset V$ is a nontrivial knot. $G \subset V$ is a compact connected oriented surface so that $L$ is a component of $\partial G$, and $\partial G-L$ (may be empty) consists of parallel essential circles on $\partial V$. Orientations on these circles are induced from the orientation on $G$, we require that these circles are parallel as oriented ones. We glue $V$ to $Y-\operatorname{int}(N(K))$, so that any component of $\partial G-L$ is null-homologous in $Y-\operatorname{int}(N(K))$. The new manifold is denoted by $Y^{*}$, and the image of $L$ in $Y^{*}$ is denoted by $K^{*}$. We then say $K^{*}$ is a satellite knot of $K$, and $K$ a companion knot of $K^{*}$. Let $p$ denote the number of components of $\partial G-L, p$ will be called the winding number of $L$ in $V$.

Moveover, if $V-L$ fibers over the circle so that $G$ is a fiber and $\chi(G)<0$, then we say $K^{*}$ is a semifibred satellite knot.

Remark 5.2 In order to avoid some trivial cases, we often need some additional condition on $G$ in the definition of satellite knot, say, incompressible in $V-L$. But this would not affect the results stated in this paper.

The classical case is $Y=Y^{*}=S^{3}$, and $V$ is a solid torus. A large number of classical satellite knots are semifibred. For example, it is well-known that cable knots are semifibred, see [15, 10.I]. A bit more work can show that if $L$ is a "homogeneous braid" in the solid torus $V$, then $K^{*}$ is semifibred (see [16]).

Our goal in this section is

Theorem 5.3 Notations as in Definition 5.1. $K^{*}$ is a semifibred satellite knot. Suppose the genera of $F, G$ are $g, h$, respectively, and the winding number is $p$. Then

$$
\widehat{H F K}\left(Y^{*}, K^{*},-(p g+h)\right) \cong \widehat{H F K}(Y, K,-g)
$$

as abelian groups.

In the case of classical knots, our result should be compared with the well-known relation for Alexander polynomial:

$$
\Delta_{K^{*}}(t)=\Delta_{K}\left(t^{p}\right) \Delta_{L}(t) .
$$

See, for example, [1].

Remark 5.4 Not many results were known previously on the knot Floer homology of satellite knots. Eftekhary computed the top filtration term for Whitehead doubles in [2]. And some terms for $(p, p n \pm 1)$ cable knots were computed by Hedden in [6]. 
Hedden and Ording also have an ongoing program to compute the Floer homology of $(1,1)$ satellite knots. Whitehead doubles are not semifibred in our sense, although $V-L$ fibers over $S^{1}$ in this case.

Construction 5.5 As the reader may have found in the last section, we have to spend most efforts on the description of the construction of a suitable Heegaard diagram, although the idea of such construction is very simple. Our construction here consists of 5 steps. The notations are as before. In this construction, we assume the monodromy of the fibred part $V-\operatorname{int}(N(L))$ is a special map $\psi$, which will be defined in Step 2.

\section{Step 0 A Heegaard splitting of $Y^{*}$}

A Heegaard splitting of $\left(Y^{*}, K^{*}\right)$ can be constructed as follows. Pick $p$ parallel copies of $F: F^{(1)}, \ldots, F^{(p)}$. Glue them to $G$, so as to get a surface $F^{*}$ of genus $p g+h$. Thicken $F^{*}$ to $F^{*} \times[0,1]$ in $Y^{*}$. Add a one handle $\mathcal{H}^{(k)}$ connecting $F^{(k)} \times 1$ and $F^{(k+1)} \times 0, k=1, \ldots, p$, here $F^{(p+1)}=F^{(1)}$. Add a 1 -handle $\mathcal{H}^{*}$ connecting $G \times 1$ to $G \times 0$, so that it is parallel to $\mathcal{H}^{(k)}$. Then add $r 1$-handles to $F^{(1)} \times 0$ in the same way as when we constructed the Heegaard splitting of $(Y, K)$ in the proof of Theorem 2.1. See Figure 7 for a schematic picture.

Now we have a Heegaard splitting $Y^{*}=U_{0}^{*} \cup U_{1}^{*}, U_{0}^{*}$ is the union of $F^{*} \times[0,1]$ and some 1-handles. In the rest of this construction, we will construct the corresponding Heegaard surface abstractly, and give the $\alpha$ and $\beta$ curves on this Heegaard surface. Hence we get a Heegaard diagram which can be fit into the Heegaard splitting we construct in Step 0.

\section{Step 1 Construct block surfaces with curves on them}

$A$ is a genus $g$ surface with boundary consisting of two circles, denoted by $\alpha_{1}, \lambda$. $A$ is basically a punctured $F \times 1$. Pick an arc $\delta$ connecting $\alpha_{1}$ to $\lambda$. Then we can choose two $2 g$-tuples of mutually disjoint proper $\operatorname{arcs}$ in $A-\delta:\left(\xi_{2}, \ldots, \xi_{2 g+1}\right)$ and $\left(\eta_{2}, \ldots, \eta_{2 g+1}\right)$, so that $\partial \xi_{i} \subset \lambda, \partial \eta_{i} \subset \alpha_{1}$. Moreover, $\eta_{i}$ is disjoint from $\xi_{j}$ when $j \neq i$, and $\eta_{i}$ intersects $\xi_{i}$ transversely in a single intersection point. The reader is referred to [13, Figure 1] for a choice of these curves.

Let $B^{*}$ be a genus $h$ surface with boundary consisting of two circles, denoted by $\alpha_{1}^{*}, \lambda^{*}$. We can choose curves $\xi_{j}^{*}, \eta_{j}^{*}, \delta^{*}$ as before. For the $\operatorname{arc} \delta^{*}$ connecting $\alpha_{1}^{*}$ to $\lambda^{*}$, we pick $p$ parallel copies, $\delta_{1}, \ldots, \delta_{p}$, lying on the same side of $\delta^{*}$. Choose a point in each $\delta_{k}$, remove a small open disk at each chosen point, then get a surface with boundary consisting of $p+2$ circles $\alpha_{1}^{*}, \lambda^{*}, \lambda^{(1)}, \ldots, \lambda^{(p)}$, called $B$. The remaining part of $\delta_{k}$ consists of two $\operatorname{arcs} \pi^{(k)}, \rho^{(k)}$, here $\pi^{(k)}$ connects $\lambda^{*}$ to $\lambda^{(k)}$. See Figure 5 for the local picture. 


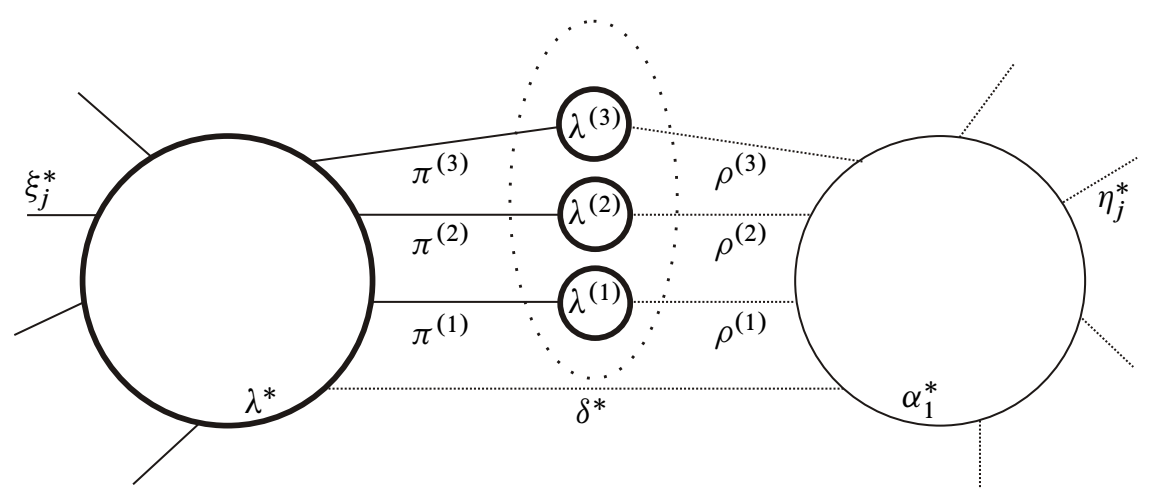

Figure 5: Local picture near $\delta^{*}$

\section{Step 2 Construct the monodromy $\psi$}

Take $G \times[0,2]$, glue the two ends together by the identity, so as to get $G \times S^{1}$. Two surfaces $G \times 0, G \times 1$ separate $G \times S^{1}$ into two parts. Choose a small disk $D_{1}$ in the interior of $G$. Denote $G \times[0,1] \cup D_{1} \times[1,2]$ by $V_{0}, G \times[1,2]-\operatorname{int}\left(D_{1}\right) \times[1,2]$ by $V_{1}$.

$\bar{B}$ denotes the copy of $B$ reflected across its boundary. Curves on $\bar{B}$ are denoted by $\bar{\xi}_{j}^{*}$, etc. Glue $B$ and $\bar{B}$ so that $\lambda^{*}, \alpha_{1}^{*}$ are identified with $\bar{\lambda}^{*}, \bar{\alpha}_{1}^{*} . B \cup \bar{B}$ can be naturally identified with the surface

$$
\left(\left(G-\operatorname{int}\left(D_{1}\right)\right) \times 1\right) \cup\left(\left(G-\operatorname{int}\left(D_{1}\right)\right) \times 0\right) \cup\left(\partial D_{1} \times[1,2]\right) \cup\left(\lambda^{*} \times[0,1]\right),
$$

where $\lambda^{*}$ is the boundary component of $G$ which corresponds to the longitude of $K^{*}$, by abuse of notation. $B \supset\left(G-\operatorname{int}\left(D_{1}\right)\right) \times 1, \bar{B} \supset\left(G-\operatorname{int}\left(D_{1}\right)\right) \times 0$.

Glue $\eta_{j}^{*}$ and $\bar{\eta}_{j}^{*}$ together to a closed curve $\beta_{j}^{*}$, glue $\xi_{j}^{*}, \bar{\xi}_{j}^{*}$ together to a closed curve $\alpha_{j}^{*}, j=2, \ldots, 2 h+1$. Glue $\delta^{*}$ and $\bar{\delta}^{*}$ together to a closed curve $\mu^{*}$. Glue $\pi^{(k)}$ and $\bar{\pi}^{(k)}$ together to an $\operatorname{arc}^{1}$. Glue $\rho^{(k)}$ and $\bar{\rho}^{(k)}$ together to an arc. We have the properties:

$(\alpha)$ The circles $\alpha_{j}^{*}(j=1,2, \ldots, 2 h+1)$ bound disks in $V_{0}$. The arc $\pi^{(k)} \cup \bar{\pi}^{(k)}$ cobounds a half-disk ${ }^{2}$ in $V_{0}$ with a vertical arc on $\lambda^{(k)} \times[0,1], k=1, \ldots, p$.

$(\beta)$ The circles $\mu^{*}, \beta_{j}^{*}(j=2, \ldots, 2 h+1)$ bound disks in $V_{1}$. The arc $\rho^{(k)} \cup \bar{\rho}^{(k)}$ cobounds a half-disk in $V_{1}$ with a vertical arc on $\lambda^{(k)} \times[1,2], k=1, \ldots, p$.

\footnotetext{
${ }^{1} \pi^{(k)}$ connects $\lambda^{*}$ to $\lambda^{(k)}, \bar{\pi}^{(k)}$ connects $\bar{\lambda}^{*}$ to $\bar{\lambda}^{(k)}$. We glued $\lambda^{*} \subset B$ to $\bar{\lambda}^{*} \subset \bar{B}$, but did not glue $\lambda^{(k)}$ to $\bar{\lambda}^{(k)}$, so $\pi^{(k)} \cup \bar{\pi}^{(k)}$ is an arc.

${ }^{2}$ Of course a half-disk is homeomorphic to a disk. We use the term "half-disk" because this disk will be part of a disk bounded by an $\alpha$ curve constructed later.
} 
As in Figure 5 , the dotted circle encloses a $p$-punctured disk $D_{2}$. There is a diffeomorphism $\psi$ of $B$, supported in $D_{2}$, sending $\lambda^{(k)}$ to $\lambda^{(k-1)}\left(\lambda^{(0)}=\lambda^{(p)}\right)$. We draw $\psi\left(\rho^{(k)}\right)$ in Figure 6.

$\psi$ can be extended by identity to a diffeomorphism of $G$, still denoted by $\psi$. Cut $G \times S^{1}$ open along $G \times 1$, reglue by $\psi$, namely, glue each point $x \in G \times(1+0)$ to $\psi(x) \in G \times(1-0)$. Now we get a surface bundle over the circle, with monodromy $\psi$. This surface bundle will serve as our $V-\operatorname{int}(N(L))$.

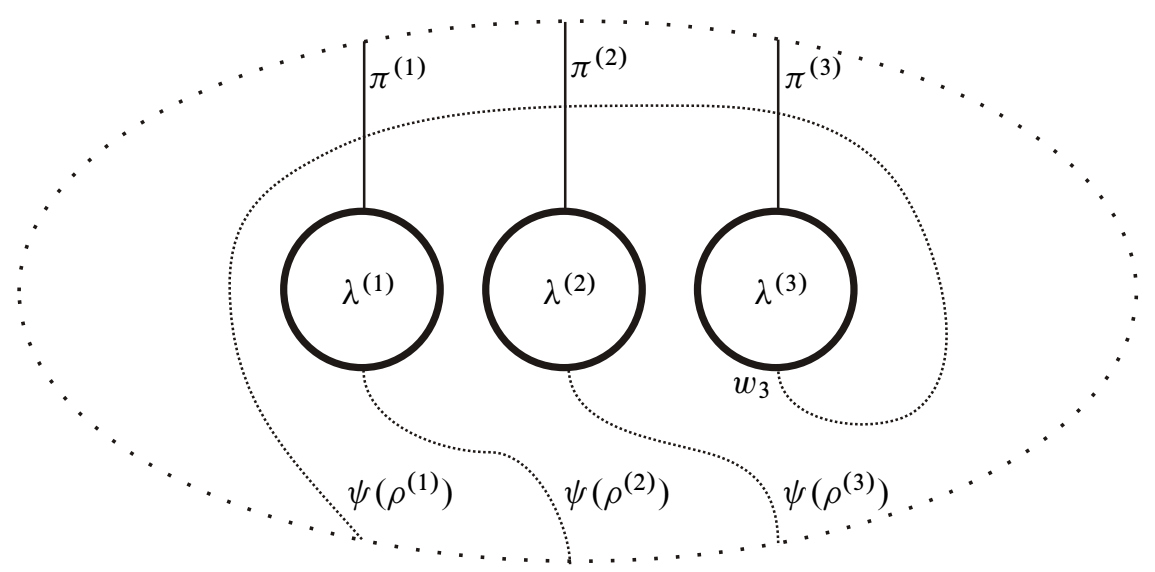

Figure 6: Local picture inside the circle

$B \cup \bar{B}$ naturally lies in $V-\operatorname{int}(N(L))$ as before, and it separates the bundle into $V_{0}$ and $V_{1}$. We will view $B \cup \bar{B}$ as living on the boundary of $V_{0}$. We again have the curves on $B \cup \bar{B}$ satisfying Properties $(\alpha),(\beta)$, except that in Property $(\beta), \psi\left(\rho^{(k)}\right) \cup \bar{\rho}^{(k)}$ cobounds a half-disk in $V_{1}$ with a vertical arc on $\lambda^{(k)} \times[1,2], k=1, \ldots, p . \partial V=T^{2}$ is the union of the annuli

$$
\lambda^{(1)} \times[0,1], \lambda^{(2)} \times[1,2], \lambda^{(2)} \times[0,1], \ldots, \lambda^{(p)} \times[0,1], \lambda^{(1)} \times[1,2] .
$$

\section{Step 3 Glue blocks together}

Take $p$ copies of $A: A^{(1)}, A^{(2)}, \ldots, A^{(p)}$, and let $\bar{A}^{(k)}$ denote the copy of $A^{(k)}$ reflected across its boundary. Curves on $A^{(1)}$ are denoted by $\xi_{i}^{(1)}, \eta_{i}^{(1)}$, etc. One may worry about the $\lambda$ curve on $A^{(k)}$, which will be called $\lambda^{(k)}$ by our convention, and this name coincides with the boundary curve $\lambda^{(k)}$ of $B$. But since we are going to identify these two curves, we do not introduce any new notation to distinguish them. Similarly, we name the curves on $\bar{A}^{(k)}$ by $\bar{\xi}_{i}^{(k)}$, etc. 


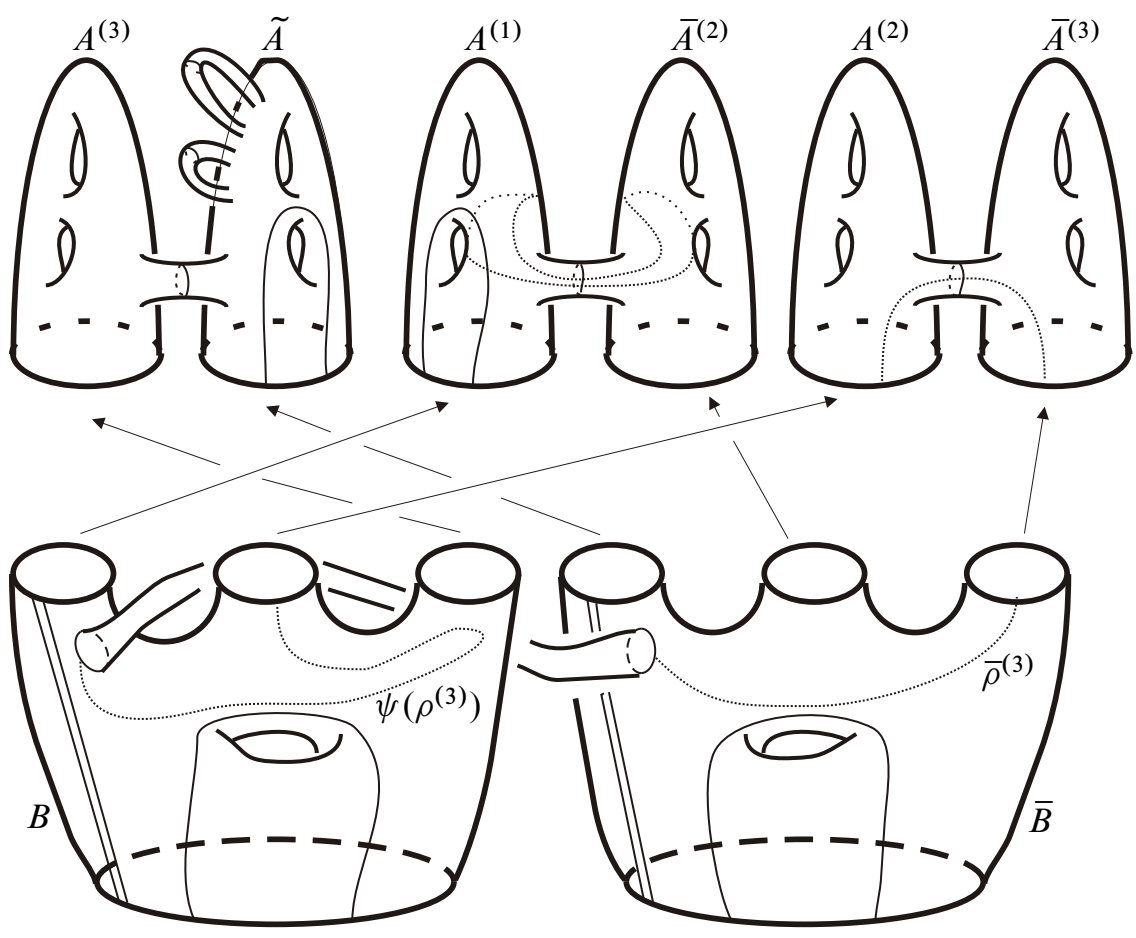

Figure 7: The Heegaard surface $\Sigma^{*}$ with some sample curves drawn

We glue $A^{(k)}$ and $\bar{A}^{(k+1)}$ so that $\alpha_{1}^{(k)}$ is identified with $\bar{\alpha}_{1}^{(k+1)}$, glue $A^{(k)}$ and $B$ along $\lambda^{(k)}$, glue $\bar{A}^{(k)}$ and $\bar{B}$ along $\bar{\lambda}^{(k)}$.

Add $r$ tubes to $\bar{A}^{(1)}$, as in Step 0. The union of the $(2 r)$-punctured $\bar{A}^{(1)}$ and the $r$ new tubes is called $\tilde{A}$. Meridians of these new tubes are called $\widetilde{\alpha}_{2 g+2}, \ldots, \widetilde{\alpha}_{2 g+1+r}$. One can choose circles $\widetilde{\beta}_{2}, \ldots, \widetilde{\beta}_{2 g+1+r}$ on $A^{(p)} \cup \tilde{A}$ as in the sutured Heegaard diagram $\left(\Sigma, \boldsymbol{\alpha}, \boldsymbol{\beta}_{\mathbf{0}} \cup\{\mu\}, w, z\right)$, so that they are disjoint from $\delta^{(p)}, \bar{\delta}^{(1)}$.

Glue $\eta_{i}^{(k)}$ and $\bar{\eta}_{i}^{(k+1)}$ together to a closed curve $\beta_{i}^{(k)}, k=1, \ldots, p-1$, $i=2, \ldots, 2 g+1$. Glue $\xi_{i}^{(k)}, \bar{\xi}_{i}^{(k)}$,two copies of $\pi^{(k)}$ and two copies of $\bar{\pi}^{(k)}$ together to a closed curve $\alpha_{i}^{(k)}, k=1,2, \ldots, p, i=2, \ldots, 2 g+1$.

Glue $\delta^{(k)}, \bar{\delta}^{(k+1)}, \psi\left(\rho^{(k+1)}\right)$ and $\bar{\rho}^{(k+1)}$ together to a closed curve $\omega^{(k)}, k=$ $1, \ldots, p$.

Step 4 A Heegaard diagram for $\left(Y^{*}, K^{*}\right)$

From last step, we have a surface

$$
\Sigma^{*}=\tilde{A} \cup A^{(1)} \cup \bar{A}^{(2)} \cup A^{(2)} \cup \cdots \cup \bar{A}^{(p)} \cup A^{(p)} \bigcup \bar{B} \cup B,
$$


with two collections of disjoint closed curves

$$
\begin{aligned}
\alpha^{*}= & \left\{\alpha_{i}^{(k)}, k=1, \ldots, p, i=2, \ldots, 2 g+1\right\} \bigcup\left\{\tilde{\alpha}_{2 g+2}, \ldots, \tilde{\alpha}_{2 g+1+r}\right\} \\
& \bigcup\left\{\alpha_{2}^{*}, \ldots, \alpha_{2 h+1}^{*}\right\} \bigcup\left\{\alpha_{1}^{*}, \alpha_{1}^{(1)}, \ldots, \alpha_{1}^{(p)}\right\}, \\
\boldsymbol{\beta}^{*}= & \left\{\beta_{i}^{(k)}, k=1, \ldots, p-1, i=2, \ldots, 2 g+1\right\} \bigcup\left\{\tilde{\beta}_{2}, \ldots, \tilde{\beta}_{2 g+1+r}\right\} \\
& \bigcup\left\{\beta_{2}^{*}, \ldots, \beta_{2 h+1}^{*}\right\} \bigcup\left\{\omega^{(1)}, \ldots, \omega^{(p)}\right\} \cup\left\{\mu^{*}\right\} .
\end{aligned}
$$

We claim that $\left(\Sigma^{*}, \boldsymbol{\alpha}^{*}, \boldsymbol{\beta}^{*}\right)$ is a Heegaard diagram for $Y^{*}$. In fact we can fit $\Sigma^{*}$ into the construction in Step 0 , so that $\bar{A}^{(k)}$ is basically $F^{(k)} \times 0$ with a hole, $A^{(k)}$ is basically $F^{(k)} \times 1$ with a hole, $\bar{B}$ is basically $G \times 0$ with a hole, $B$ is basically $G \times 1$ with a hole. Now the proof of our claim is as easy as ABC, it is easy to check the following:

(A) $\operatorname{genus}\left(\Sigma^{*}\right)=\left|\boldsymbol{\alpha}^{*}\right|=\left|\boldsymbol{\beta}^{*}\right|=2 p g+2 h+r+p+1$.

(B) Curves in $\alpha^{*}$ bound disks in $U_{0}^{*}$, curves in $\beta^{*}$ bound disks in $U_{1}^{*}$.

(For example, in order to check that $\alpha_{i}^{(k)}$ bounds a disk in $U_{0}^{*}$, we recall that $\alpha_{i}^{(k)}$ is the union of $\xi_{i}^{(k)}, \bar{\xi}_{i}^{(k)}$,two copies of $\pi^{(k)}$ and two copies of $\bar{\pi}^{(k)} \cdot \xi_{i}^{(k)}$ and $\bar{\xi}_{i}^{(k)}$ are two parallel sides of a rectangle between $A^{(k)}$ and $\bar{A}^{(k)}, \pi^{(k)} \cup \bar{\pi}^{(k)}$ cobounds a half-disk in $V_{0}$ with a vertical $\operatorname{arc}$ on $\lambda^{(k)} \times[0,1]$, (see Property $(\alpha)$ in Step 2,) the union of the rectangle and two copies of the half-disk is a disk bounded by $\alpha_{i}^{(k)}$ in $U_{0}^{*}$.)

(C) $\Sigma^{*}-\boldsymbol{\alpha}^{*}$ is connected, $\Sigma^{*}-\boldsymbol{\beta}^{*}$ is connected.

Pick two points $w^{*}, z^{*}$ near $\lambda^{*} \cap \mu^{*}$ as understood. Then $\left(\Sigma^{*}, \boldsymbol{\alpha}^{*}, \boldsymbol{\beta}^{*}, w^{*}, z^{*}\right)$ is a double pointed diagram for $\left(Y^{*}, K^{*}\right)$.

Strictly speaking, the Heegaard diagram constructed above is not a sutured Heegaard diagram. In Definition 2.2, in a sutured Heegaard diagram, there is a subsurface $\mathcal{P}$ bounded by 2 curves $\lambda$ and $\alpha_{1}$. In our diagram here, the corresponding subsurface

$$
\mathcal{P}^{*}=A^{(1)} \cup \cdots \cup A^{(p)} \cup B
$$

has $p+2$ boundary components $\lambda^{*}, \alpha_{1}^{*}, \alpha_{1}^{(1)}, \ldots, \alpha_{1}^{(p)}$.

However, we can still handle this diagram by the same method we used in Section 3. (We could have extended Definition 2.2 to the case of more boundary components, but we would rather choose the current version for simplicity.)

We claim that the generators of $\widehat{C F K}\left(Y^{*}, K^{*},-(p g+h)\right)$ lie outside the interior of $\mathcal{P}^{*}$. In fact $\chi\left(\mathcal{P}^{*}\right)=-2(p g+h)-p$, and we have to choose points on $\alpha_{1}^{(1)}, \ldots, \alpha_{1}^{(p)}$, 
where the local multiplicity is $\frac{1}{2}$. Argue as in Step 3 of Theorem 2.1, we can prove the claim. (We cheat a little bit here, since the diagram is not known to be weakly admissible now.)

By the construction of the Heegaard diagram for $\left(Y^{*}, K^{*}\right)$, there is only one choice for the generators of $\widehat{C F K}\left(Y^{*}, K^{*},-(p g+h)\right)$ outside $\widetilde{A}$. Hence the domains of the holomorphic disks corresponding to the boundary map will restrict to relative periodic domains outside of $\tilde{A}$. We want to show that these holomorphic disks are supported inside $A^{(p)} \cup \tilde{A}$, hence they are in one-to-one correspondence with the holomorphic disks for $\widehat{C F K}(Y, K,-g)$. The basic method is also winding.

Proof of Theorem 5.3 Suppose the monodromy of $V-\operatorname{int}(N(L))$ is $\varphi$. Since the fiber $G$ is connected, and has $p$ parallel components on $\partial V, \varphi$ must permute the $p$ components cyclically. Without loss of generality, we can assume $\varphi$ sends $\lambda^{(k)}$ to $\lambda^{(k-1)}$. Hence $\varphi \circ \psi^{-1}$ is isotopic to a diffeomorphism of $G$, which restricts to identity on $\partial G$. Here $\psi$ is the monodromy constructed in Step 2 of the previous construction. Now we change the monodromy of the fibred part to $\psi$, so as to get a new knot in a new manifold. The new pair is still denoted by $\left(Y^{*}, K^{*}\right)$. Proposition 3.5 says that we only need to prove our theorem for this new knot. The construction before gives a Heegaard diagram for $\left(Y^{*}, K^{*}\right)$.

In $B$, we can choose $2 h$ simple closed curves $\tau_{2}^{*}, \ldots, \tau_{2 h+1}^{*}$, such that they are disjoint from $\delta^{*}, \rho^{(k)}, \pi^{(k)}, \xi_{j}^{*}$, except that $\tau_{j}^{*}$ intersects $\xi_{j}^{*}$ transversely in a single intersection point.

Choose an arc $a \subset \lambda^{*}$, such that $a$ intersects $\delta^{*}$ and all $\xi_{j}^{*}$ 's, but $a$ is disjoint from $\pi^{(1)}, \ldots, \pi^{(p)}$. Let $\mathcal{U}$ be a small neighborhood of $a$. Wind $\xi_{j}^{*}$ 's along $\tau_{j}^{*}$ 's sufficiently many times, and apply Lemma 3.2, we find that if $\mathcal{D}^{*}$ is a nonnegative relative periodic domain in $B$, then $\partial \mathcal{D}^{*}$ does not pass through $\xi_{j}^{*}$ 's. Moreover, the local multiplicity of $\mathcal{D}^{*}$ is 0 at a point $w_{p} \in \lambda^{(p)}$ near $\psi\left(\rho^{(1)}\right) \cap \lambda^{(p)}$ (see Figure 6).

Choose an $\operatorname{arc} b \subset \lambda^{(p)}, b$ intersects $\pi^{(p)}, b \ni w_{p}$, but $b$ is disjoint from $\psi\left(\rho^{(1)}\right)$. Let $\mathcal{V}$ be a neighborhood of $b, w_{p}$ is the base point. Apply Lemma 3.2 to the surface $A^{(p)}$, we get the following conclusion: if $\mathcal{D}^{(p)}$ is a nonnegative relative periodic domain in $A^{(p)}$, then $\partial \mathcal{D}^{(p)}$ does not pass through $\xi_{i}^{(p)}$ 's. Moreover, $\partial \mathcal{D}^{(p)}$ does not pass through $\delta^{(p)}$, since the $\beta$ curves are disjoint from $\lambda^{(p)}$ while $\delta^{(p)}$ intersects $\lambda^{(p)}$ in exactly one point.

A consequence of the previous paragraph is: if $\mathcal{D}^{\prime}$ is a nonnegative relative periodic domain of $\Sigma-\tilde{A}$, then $\partial \mathcal{D}^{\prime}$ does not pass through $\bar{\xi}_{i}^{(p)}$ 's. Moreover, $\partial \mathcal{D}^{\prime}$ does not pass through the rest of the curves in $\bar{A}^{(p)}$, because $\bar{\eta}_{2}^{(p)}, \ldots, \bar{\eta}_{2 g+1}^{(p)}, \bar{\delta}^{(p)}$ are linearly 
independent in $\mathrm{H}_{1}\left(\bar{A}^{(p)}, \partial \bar{A}^{(p)}\right)$, and $\alpha^{(p-1)}$ itself can not bound a relative periodic domain in $\bar{A}^{(p)}$. Of course, here we can choose a point near $\bar{\delta}^{(p)} \cap \bar{\lambda}^{(p)}$ as the base point in $\bar{A}^{(p)}$.

We can go on with the above argument applied to $A^{(p-1)}, \bar{A}^{(p-1)}, \ldots, A^{(1)}, B, \bar{B}$ inductively, to conclude that the local multiplicity of $\mathcal{D}^{\prime}$ is 0 in these subsurfaces.

We also wind $\tilde{\alpha}_{2 g+2}, \ldots, \widetilde{\alpha}_{2 g+1+r}$ in $\tilde{A}$, as in the proof of Proposition 3.3. Hence the new diagram $\left(\Sigma^{*}, \boldsymbol{\alpha}^{* \prime}, \boldsymbol{\beta}^{*}, w^{*}, z^{*}\right)$ after winding is weakly admissible. Moreover, the domains of the holomorphic disks corresponding to the boundary map will restrict to relative periodic domains in $\Sigma-\tilde{A}$, so the holomorphic disks for $\widehat{C F K}\left(Y^{*}, K^{*},-(p g+h)\right)$ are supported inside $A^{(p)} \cup \widetilde{A}$. Hence they are in oneto-one correspondence with the holomorphic disks for $\widehat{C F K}(Y, K,-g)$. Then our desired result holds.

\section{References}

[1] G Burde, H Zieschang, Knots, de Gruyter Studies in Mathematics 5, Walter de Gruyter \& Co., Berlin (2003) MR1959408

[2] E Eftekhary, Longitude Floer homology and the Whitehead double, Algebr. Geom. Topol. 5 (2005) 1389-1418 MR2171814

[3] D Gabai, The Murasugi sum is a natural geometric operation, from: "Low-dimensional topology (San Francisco, Calif., 1981)", Contemp. Math. 20, Amer. Math. Soc., Providence, RI (1983) 131-143 MR718138

[4] D Gabai, The Murasugi sum is a natural geometric operation. II, from: "Combinatorial methods in topology and algebraic geometry (Rochester, N.Y., 1982)", Contemp. Math. 44, Amer. Math. Soc., Providence, RI (1985) 93-100 MR813105

[5] D Gabai, Foliations and the topology of 3-manifolds. III, J. Differential Geom. 26 (1987) 479-536 MR910018

[6] M Hedden, On knot Floer homology and cabling, Algebr. Geom. Topol. 5 (2005) 1197-1222 MR2171808

[7] Y Ni, A note on knot Floer homology of links arXiv:math.GT/0506208

[8] P Ozsváth, Z Szabó, Heegaard diagrams and holomorphic disks, from: "Different faces of geometry”, Int. Math. Ser. (N. Y.), Kluwer/Plenum, New York (2004) 301-348 MR2102999

[9] P Ozsváth, Z Szabó, Holomorphic disks and genus bounds, Geom. Topol. 8 (2004) 311-334 MR2023281

[10] P Ozsváth, Z Szabó, Holomorphic disks and knot invariants, Adv. Math. 186 (2004) 58-116 MR2065507 
[11] P Ozsváth, Z Szabó, Holomorphic disks and three-manifold invariants: properties and applications, Ann. of Math. (2) 159 (2004) 1159-1245 MR2113020

[12] P Ozsváth, Z Szabó, Holomorphic disks and topological invariants for closed threemanifolds, Ann. of Math. (2) 159 (2004) 1027-1158 MR2113019

[13] P Ozsváth, Z Szabó, Heegaard Floer homology and contact structures, Duke Math. J. 129 (2005) 39-61 MR2153455

[14] J Rasmussen, Floer homology and knot complements, PhD thesis, Harvard (2003) arXiv:math.GT/0306378

[15] D Rolfsen, Knots and links, Mathematics Lecture Series 7, Publish or Perish Inc., Houston, TX (1990) MR1277811 Corrected reprint of the 1976 original

[16] J R Stallings, Constructions of fibred knots and links, from: "Algebraic and geometric topology (Proc. Sympos. Pure Math., Stanford Univ., Stanford, Calif., 1976), Part 2", Proc. Sympos. Pure Math., XXXII, Amer. Math. Soc., Providence, R.I. (1978) 55-60 MR520522

Department of Mathematics, Princeton University, Princeton, NJ 08544, USA

yni@math.princeton.edu

Received: 22 July 2005 Revised: 13 January 2006 
